\title{
An example of non-existence of plane-like minimizers for an almost-periodic Ising system
}

\author{
Andrea Braides \\ Dipartimento di Matematica, Università di Roma Tor Vergata \\ via della ricerca scientifica 1, 00133 Roma, Italy
}

\section{Introduction}

The homogenization of ferromagnetic spin systems in deterministic or random environments [3], as well as in some aperiodic settings [4], has been carried over in analogy with the homogenization of surface energies [2]. The computation of an effective surface energy for such systems relies on the characterization of those ground states that follow a planar interface, and the related homogenization formulas. For systems with periodic coefficients it has been shown that the energy of such ground states can be confined on a strip of finite width around a plane (plane-like minimizers) [5]. In this paper we show that this is not the case if the coefficients are uniformly almost periodic by giving an explicit two-dimensional example where there is no ground state confined on a strip. In this example the coefficents are the uniform limit of periodic coefficients (with increasing period).

\section{Setting of the problem}

We consider a discrete system of nearest-neighbour interactions in dimension two with coefficients $c_{i j} \geq c>0, i, j \in \mathbb{Z}^{2}$. The corresponding ferromagnetic spin energy is

$$
F(u)=\sum_{i j} c_{i j}\left(u_{i}-u_{j}\right)^{2}
$$

where $u: \mathbb{Z}^{2} \rightarrow\{-1,1\}, u_{i}=u(i)$, and the sum runs over the set of nearest neighbours or bonds in $\mathbb{Z}^{2}$, which is denoted by

$$
\mathcal{Z}=\left\{(i, j) \in \mathbb{Z}^{2} \times \mathbb{Z}^{2}:|i-j|=1\right\} .
$$

Such energies correspond to inhomogeneous surface energies on the continuum $[1,3]$. 
Definition 1 We say that $u$ is a ground state if we have

$$
\sum_{i j} c_{i j}\left(\left(u_{i}-u_{j}\right)^{2}-\left(v_{i}-v_{j}\right)^{2}\right) \leq 0
$$

for all $v$ such that $v_{i}=u_{i}$ except for a finite number of indices (so that actually the sum runs over a finite set).

Definition 2 We say that $u$ is a plane-like ground state or plane-like minimizer for $F$ in the direction $\nu$ if $u$ is a ground state and there exists a number $M$ such that (up to a change of sign of all values of $u$ ) we have

$$
u_{i}= \pm 1 \quad \text { if } \pm\langle i, \nu\rangle \geq M
$$

The relevance of this definition lies in a result by Caffarelli and de la Llave, who proved that if $c_{i j}$ is periodic then for all directions $\nu$ there exists a plane-like minimizer of $F$ in the direction $\nu[5]$.

If we identify the function $u$ with its piecewise-constant interpolation, then being a plane-like minimizer can be interpreted as the property that the interface $\partial\{u=1\}$ lies in a strip around a line (or a hyperplane in higher dimension, whence the name plane-like minimizer). Note that this interface cannot be periodic if $\nu$ is an 'irrational' direction.

\section{The example}

This section is devoted to an example of uniformly almost-periodic coefficients $c_{i j}$ such that there exits plane-like minimizer for the corresponding $F$ for all directions $\nu$.

We consider the following nested sets: for $n \geq 1$ we define

$$
B_{n}=\left\{(i, j) \in \mathcal{Z}: \frac{i_{1}+j_{1}}{2} \text { or } \frac{i_{2}+j_{2}}{2} \in \frac{1}{2}+2 \cdot 3^{n}+4 \cdot 3^{n} \mathbb{Z}\right\}
$$

Since $2 \cdot 3^{n+1}+4 \cdot 3^{n+1} \mathbb{Z} \subset 2 \cdot 3^{n}+4 \cdot 3^{n} \mathbb{Z}$ we have $B_{n+1} \subset B_{n}$. We set $B_{0}=\mathcal{Z}$.

For all $i, j \in \mathbb{Z}^{2}$ with $|i-j|=1$ we set

$$
c_{i j}=\frac{1}{2}+\frac{1}{2^{n}} \text { if }(i, j) \in B_{n} \backslash B_{n+1}, \quad n=0,1, \ldots
$$

Remark 3 (almost-periodicity) Note that the coefficients $c_{i j}^{n}$ defined by

$$
c_{i j}^{n}=\max \left\{c_{i j}, \frac{1}{2}+\frac{1}{2^{n}}\right\}
$$

are $4 \cdot 3^{n}$-periodic and converge uniformly to $c_{i j}$ on $\mathcal{Z}$. Hence, the system of coefficients $c_{i j}$ is uniformly almost periodic; more precisely, it is the uniform limit of a family of periodic coefficients of increasing periods. 
Remark 4 (homogenizability) Note that the set of coefficients $c_{i j}$ is homogenizable (in the terminology of [3]). If we define the family of energies

$$
F_{\varepsilon}(u)=\sum_{i j} \varepsilon c_{i j}\left(u_{i}-u_{j}\right)^{2} \quad \text { if } u: \varepsilon \mathbb{Z}^{2} \rightarrow\{-1,1\}
$$

where $u_{i}=u(\varepsilon i)$, then, upon identifying each $u$ with its piecewise-constant interpolation as a $L^{1}$-function, $F_{\varepsilon} \Gamma$-converge to the energy

$$
F_{0}(u)=4 \int_{\partial\{u=1\}}\left(\left|\nu_{1}\right|+\left|\nu_{2}\right|\right) d \mathcal{H}^{1} \quad \text { if } u \in B V\left(\mathbb{R}^{2} ;\{-1,1\}\right)
$$

where $\partial\{u=1\}$ is understood as the reduced boundary of the set $\{u=1\}$ and $\nu$ its measure-theoretical normal.

This can be proved using the results in [3], or directly by comparison, on one side remarking that, using that $c_{i j} \geq 1 / 2$ for all $i$ and $j$, we have

$$
F_{\varepsilon}(u) \geq \frac{1}{2} \sum_{i j} \varepsilon\left(u_{i}-u_{j}\right)^{2} \quad \text { if } u: \varepsilon \mathbb{Z}^{2} \rightarrow\{-1,1\}
$$

and the $\Gamma$-limit of the energies of this right-hand side is $F_{0}$ by [1]. On the other side, by the remark above, for all $u$ we can find a sequences of functions $\left\{u_{\varepsilon}\right\}$ converging to $u$ and such that

$$
F_{\varepsilon}\left(u_{\varepsilon}\right) \leq\left(\frac{1}{2}+\frac{1}{2^{n}}\right) \sum_{i j} \varepsilon\left(\left(u_{\varepsilon}\right)_{i}-\left(u_{\varepsilon}\right)_{j}\right)^{2} \leq\left(4+\frac{8}{2^{n}}\right) \mathcal{H}^{1}(\partial\{u=1\})
$$

(the factor 8 comes from the fact that each nearest-neighbour pair is accounted for twice, and that $\left(\left(u_{\varepsilon}\right)_{i}-\left(u_{\varepsilon}\right)_{j}\right)^{2}=4$ for non-zero interactions).

We now show that there exists no plane-like minimizer for the energy $F$ in any direction $\nu$. We first consider the case when $\nu$ is not any coordinate direction. By symmetry it is sufficient to consider the case

$$
\nu_{1}<0, \quad 0<\nu_{2} \leq-\nu_{1}
$$

i.e., the direction of the strip

$$
S_{\nu}^{M}:=\left\{x \in \mathbb{R}^{2}:\langle x, \nu\rangle \mid \leq M\right\}
$$

is increasing and at an angle not less than 45 degrees.

Suppose that such a plane-like minimizer $u$ existed, and let $\nu, M$ be given by its definition. Up to changing the sign to $u$ we may suppose that (3). 
With fixed $n$, let $k_{n}$ be the minimal $k$ such that the horizontal line

$$
x_{2}=\frac{1}{2}+2 \cdot 3^{n}+4 \cdot 3^{n} k
$$

intersects

$$
S_{\nu}^{M} \cap\left\{\left(x_{1}, x_{2}\right): x_{1}>\frac{1}{2}+2 \cdot 3^{n}\right\}
$$

i.e., the intersection of the strip with the half-plane on the right-hand side of the first vertical line of $B_{n}$.

We consider the function $v$ defined as

$$
v_{i}= \begin{cases}1 \quad & \text { if } i_{1}<\frac{1}{2}+2 \cdot 3^{n} \text { and } i_{2}>\frac{1}{2}-2 \cdot 3^{n}+4 \cdot 3^{n} k_{n} \\ -1 & \text { if } i_{1}>\frac{1}{2}+2 \cdot 3^{n} \text { and } i_{2}<\frac{1}{2}+2 \cdot 3^{n}+4 \cdot 3^{n} k_{n} \\ u_{i} & \text { otherwise. }\end{cases}
$$

Note that by $(3)$ the set $\left\{I: u_{i} \neq v_{i}\right\}$ is finite and contained in the horizontal strip defined by

$$
S_{n}=\left\{\left(x_{1}, x_{2}\right): \frac{1}{2}-2 \cdot 3^{n}+4 \cdot 3^{n} k_{n}<x_{2}<\frac{1}{2}+2 \cdot 3^{n}+4 \cdot 3^{n} k_{n}\right\} .
$$

If we identify the discrete function $u$ with its piecewise-constant interpolation

$$
u(x)=u\left(\left\lfloor x_{1}-\frac{1}{2}\right\rfloor,\left\lfloor x_{2}-\frac{1}{2}\right\rfloor\right)
$$

then $u$ can be pictured through the interface $\partial\{u=1\}$, and likewise $v$. In Fig. 1 the solid line represents the interface $\partial\{v=1\}$ and the dotted line the part of the interface $\partial\{u=1\}$ not included in $\partial\{v=1\}$. The vertical and horizontal lines represent the interactions in $B_{n}$

We now compute the variation of the energy

$$
\sum_{i j} c_{i j}\left(\left(u_{i}-u_{j}\right)^{2}-\left(v_{i}-v_{j}\right)^{2}\right),
$$

which we estimate separately on the sets

$$
\begin{gathered}
I_{1}=\left\{(i, j): \frac{i_{2}+j_{2}}{2}=\frac{1}{2}-2 \cdot 3^{n}+4 \cdot 3^{n} k_{n},\left(i_{1}, j_{1}\right) \text { or }\left(i_{2}, j_{2}\right) \in S_{\nu}^{M}\right\} \\
I_{2}=\left\{(i, j): \frac{i_{2}+j_{2}}{2}=\frac{1}{2}+2 \cdot 3^{n}+4 \cdot 3^{n} k_{n},\left(i_{1}, j_{1}\right) \text { or }\left(i_{2}, j_{2}\right) \in S_{\nu}^{M}\right\} \\
I_{3}=\left\{(i, j): \frac{i_{1}+j_{1}}{2}=\frac{1}{2}+2 \cdot 3^{n},\left(i_{1}, j_{1}\right) \text { or }\left(i_{2}, j_{2}\right) \in S_{\nu}^{M}\right\}
\end{gathered}
$$




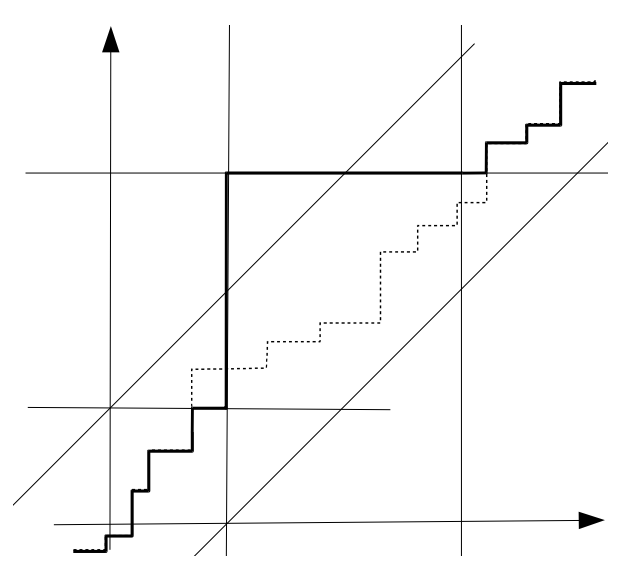

Figure 1: Construction of a competitor $v$ (oblique case)

$$
\begin{gathered}
I_{u}=\left\{(i, j): i, j \in S_{\nu}^{M} \cap S_{n},(i, j) \notin I_{1} \cup I_{2} \cup I_{3}\right\} \\
I_{v}=\left\{(i, j): v_{i} \neq v_{j},(i, j) \notin I_{1} \cup I_{2} \cup I_{3} ; i \text { or } j \in S_{n}\right\} .
\end{gathered}
$$

Note that outside the union of these sets $u_{i}=v_{i}$ and $u_{j}=v_{j}$; note moreover that

$$
\begin{gathered}
c_{i j} \leq \frac{1}{2}+\frac{1}{2^{n}} \text { on } I_{1} \cup I_{2} \cup I_{3} \cup I_{v} \\
c_{i j} \geq \frac{1}{2}+\frac{1}{2^{n-1}} \text { on } I_{u} .
\end{gathered}
$$

Up to taking a larger $M$ we can suppose that

- $u_{i}$ has the same value of $u_{j}$ if $(i, j) \in \mathcal{Z}$ and $i \notin S_{\nu}^{M}$ (i.e., we have no interactions on the boundary of $\left.S_{\nu}^{M}\right)$;

- the number of interactions in $I_{1}$ and $I_{2}$ (respectively, $I_{3}$ ) can be estimated by $4 M /\left|\nu_{1}\right|$ (respectively, by $4 M /\left|\nu_{2}\right|$ ). Note that $2 M /\left|\nu_{1}\right|$ (respectively, $2 M /\left|\nu_{2}\right|$ ) is the length of the intersection of an horizontal (respectively, vertical) line with $S_{\nu}^{M}$.

We then have

$$
\begin{aligned}
\sum_{(i, j) \in I_{1} \cup I_{2}} c_{i j}\left(\left(u_{i}-u_{j}\right)^{2}-\left(v_{i}-v_{j}\right)^{2}\right) & \geq-\sum_{(i, j) \in I_{1} \cup I_{2}} c_{i j}\left(v_{i}-v_{j}\right)^{2} \\
& \geq-\left(\frac{1}{2}+\frac{1}{2^{n}}\right) 4 \cdot \frac{8 M}{\left|\nu_{1}\right|} \\
\sum_{(i, j) \in I_{3}} c_{i j}\left(\left(u_{i}-u_{j}\right)^{2}-\left(v_{i}-v_{j}\right)^{2}\right) & \geq-\sum_{(i, j) \in I_{3}} c_{i j}\left(v_{i}-v_{j}\right)^{2} \\
& \geq-\left(\frac{1}{2}+\frac{1}{2^{n}}\right) 4 \cdot \frac{4 M}{\left|\nu_{2}\right|}
\end{aligned}
$$




$$
\begin{aligned}
\sum_{(i, j) \in I_{u}} c_{i j}\left(\left(u_{i}-u_{j}\right)^{2}-\left(v_{i}-v_{j}\right)^{2}\right) & =\sum_{(i, j) \in I_{u}} c_{i j}\left(u_{i}-u_{j}\right)^{2} \\
& \geq\left(\frac{1}{2}+\frac{1}{2^{n-1}}\right)\left(4 \cdot 3^{n}+4 \cdot 3^{n} \frac{\left|\nu_{2}\right|}{\left|\nu_{1}\right|}+\frac{8 M}{\left|\nu_{2}\right|}\right) \\
\sum_{(i, j) \in I_{v}} c_{i j}\left(\left(u_{i}-u_{j}\right)^{2}-\left(v_{i}-v_{j}\right)^{2}\right) & =-\sum_{(i, j) \in I_{v}} c_{i j}\left(v_{i}-v_{j}\right)^{2} \\
\geq & -\left(\frac{1}{2}+\frac{1}{2^{n}}\right)\left(4 \cdot 3^{n}-\frac{4 M}{\left|\nu_{2}\right|}+4 \cdot 3^{n} \frac{\left|\nu_{2}\right|}{\left|\nu_{1}\right|}-\frac{8 M}{\left|\nu_{2}\right|}\right) .
\end{aligned}
$$

From estimates (7)-(10) we obtain

$$
\sum_{(i, j) \in I_{u}} c_{i j}\left(\left(u_{i}-u_{j}\right)^{2}-\left(v_{i}-v_{j}\right)^{2}\right) \geq \frac{3^{n}}{2^{n}} \cdot 4\left(1+\frac{\left|\nu_{2}\right|}{\left|\nu_{1}\right|}\right)-32 M\left(\frac{1}{\left|\nu_{1}\right|}+\frac{1}{\left|\nu_{2}\right|}\right) .
$$

If $n$ is large enough the right-hand side of this expression is positive, contradicting (2).

It remains the case when $\nu_{1} \nu_{2}=0$. By symmetry it suffices to consider the case $\nu_{1}=0$; i.e., when we suppose that $u$ is a ground state such that there exists $M$ such that

$$
u_{i}=1 \quad \text { if } i_{2}>M, \quad u_{i}=-1 \quad \text { if } i_{2}<-M .
$$

Let $S_{M}=\left\{x:\left|x_{1}\right| \leq M\right\}$, and let $n$ be such that

$$
2 \cdot 3^{n}>M+2 \text {. }
$$

In this case there is no pair $(i, j) \in B_{n} \cap S_{M}$ with $i_{2}=j_{2}$ (i.e., there is no 'horizontal' bond in $B_{n}$ lying in the strip $S_{M}$ ).

With fixed $k \in \mathbb{N}$ we define a test function $v$ as follows:

$$
v_{i}= \begin{cases}-1 & \text { if } 2 \cdot 3^{n}<i_{1} \leq 2(1+2 k) 3^{n}, i_{2}<2 \cdot 3^{n} \\ u_{i} & \text { otherwise }\end{cases}
$$

We can picture the functions $u$ and $v$ through the interfaces related to their piecewiseconstant interpolations as done in the oblique case above. In Fig.2 the boldface solid line represents the interface related to $v$, the boldface dotted line represents the part of the interface related to $u$ not included in that of $v$, the other solid lines represent the location of the bonds in $B_{n}$.

Let

$$
I_{1}=\left\{(i, j) \in \mathcal{Z} \cap B_{n}: \frac{i_{1}+j_{1}}{2}=\frac{1}{2}+2 \cdot 3^{n}\right\}
$$




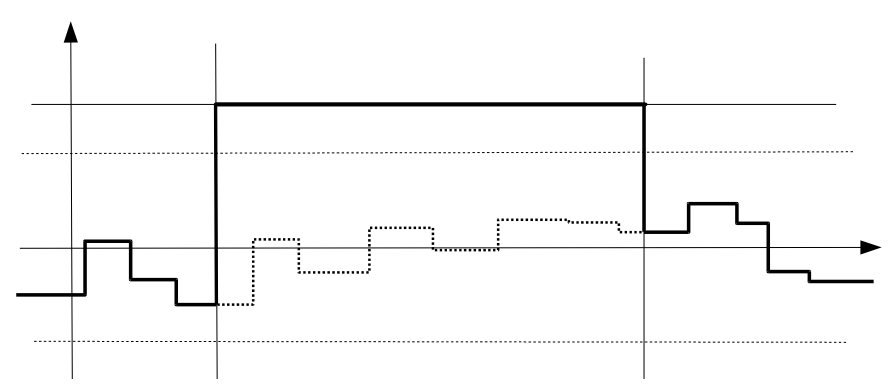

Figure 2: Construction of a competitor $v$ (horizontal case)

$$
\begin{gathered}
I_{2}=\left\{(i, j) \in \mathcal{Z} \cap B_{n}: \frac{i_{1}+j_{1}}{2}=\frac{1}{2}+2(1+2 k) 3^{n}\right\} \\
I_{3}=\left\{(i, j) \in \mathcal{Z} \cap B_{n}: \frac{i_{2}+j_{2}}{2}=\frac{1}{2}+2 \cdot 3^{n}\right\} \\
I_{u}=\left\{(i, j) \in \mathcal{Z} \cap B_{n}: i, j \in S_{M}, 2 \cdot 3^{n}<\min \left\{i_{1}, j_{1}\right\}, \max \left\{i_{1}, j_{1}\right\} \leq 2(1+2 k) 3^{n}\right\}
\end{gathered}
$$

We can then estimate

$$
\begin{aligned}
& \sum_{i j} c_{i j}\left(\left(u_{i}-u_{j}\right)^{2}-\left(v_{i}-v_{j}\right)^{2}\right) \\
= & \sum_{(i, j) \in I_{1} \cup I_{2} \cup I_{3} \cup I_{u}} c_{i j}\left(\left(u_{i}-u_{j}\right)^{2}-\left(v_{i}-v_{j}\right)^{2}\right) \\
\geq & -\sum_{(i, j) \in I_{1} \cup I_{2} \cup I_{3}}\left(\frac{1}{2}+\frac{1}{2^{n}}\right)\left(v_{i}-v_{j}\right)^{2}+\sum_{(i, j) \in I_{u}}\left(\frac{1}{2}+\frac{1}{2^{n-1}}\right)\left(u_{i}-u_{j}\right)^{2} \\
\geq & -8\left(\frac{1}{2}+\frac{1}{2^{n}}\right) \#\left(I_{1} \cup I_{2} \cup I_{3}\right)+8\left(\frac{1}{2}+\frac{1}{2^{n-1}}\right) \# I_{3},
\end{aligned}
$$

where in the estimate for the sum on $I_{u}$ we have taken into account only horizontal bonds where $u_{i} \neq u_{j}$ (whose number is greater than \#I $I_{3}$ ). We can then estimate

$$
\begin{aligned}
\sum_{i j} c_{i j}\left(\left(u_{i}-u_{j}\right)^{2}-\left(v_{i}-v_{j}\right)^{2}\right) & \geq-8\left(\frac{1}{2}+\frac{1}{2^{n}}\right)\left(8 \cdot 3^{n}+4 k 3^{n}\right)+8\left(\frac{1}{2}+\frac{1}{2^{n-1}}\right) 4 k 3^{n} \\
& \geq-64 \cdot 3^{n}+4 k \frac{3^{n}}{2^{n}}
\end{aligned}
$$

By taking $k$ large enough (recall that now $n$ is fixed by (12)) the last expression is positive, again contradicting (2). 


\section{Acknowledgments}

The example in this paper has been constructed after a question by Matteo Novaga at a Oberwolfach Workshop on "Interplay of Analysis and Probability in Physics" in January 2012 .

\section{References}

[1] R. Alicandro, A. Braides, M. Cicalese. Phase and anti-phase boundaries in binary discrete systems: a variational viewpoint. Netw. Heterog. Media 1 (2006), 85-107

[2] L. Ambrosio and A. Braides. Functionals defined on partitions of sets of finite perimeter, II: semicontinuity, relaxation and homogenization. J.Math. Pures. Appl. 69 (1990), $307-333$.

[3] A. Braides and A. Piatnitski. Homogenization of surface and length energies for spin systems. J. Funct. Anal. 264 (2013), 1296-1328

[4] A. Braides and M. Solci. Interfacial energies on Penrose lattices. M3AS 21 (2011), $1193-1210$

[5] L.A. Caffarelli and R. de la Llave. Interfaces of Ground States in Ising Models with Periodic Coefficients. J. Stat. Phys. 118 (2005), 687-719 